\title{
Der Selbstmanagement-Ansatz im Coaching - Modelle, Methoden, Anwendungsfelder
}

\author{
Karl Kubowitsch (als Gastherausgeber)
}

Online publiziert: 16. Januar 2015

(C) Springer Fachmedien Wiesbaden 2015

Diese OSC Ausgabe widmet sich dem „Selbstmanagement-Ansatz im Coaching“. Als offene verhaltenstherapeutische Konzeption hat sich die Selbstmanagement-Therapie in der Klinischen Psychologie seit den 1990er Jahren etabliert, und inzwischen liegen inhaltlich adaptierte wie auch empirisch exemplarisch überprüfte Modelle und Methoden für den Bereich des Coachings vor. Im Mittelpunkt steht hierbei, mit den Worten von Astrid Schreyögg (2012, S. 64), ein Prozess der kontinuierlichen Selbstsozialisierung, dessen Ablauf und Methodik klassischen Managementfunktionen entsprechen.

Welche Relevanz kommt einem solchen Ansatz für die Coachingpraxis zu? Zur Verbreitung unterschiedlicher theoretischer Orientierungen im Coaching in Deutschland liegen, wenn schon keine repräsentativen Studien, so doch wenigstens Indizien vor. Die Übersicht zu aktuell mehr als 300 Coachingausbildungen auf der Online-Plattform www.coaching-index.de (2014) lässt, bei allen Verweisen auf ein integratives Verständnis der Maßnahmenform, eine gewisse Dominanz systemisch ausgerichteter Angebote erahnen. Ein ähnliches Bild zeichnen bereits Umfragestudien aus den 1990er Jahren bei Coaches zu deren bevorzugten Techniken (Stahl und Marlinghaus 2000), denen zufolge systemisches Coaching an erster Stelle rangiert, gefolgt von Neurolinguistischem Programmieren, Gestalttherapie, Transaktionsanalyse sowie Psychoanalyse; verhaltensorientierte Konzepte schaffen es hier immerhin auf Platz sechs. Wenn also nicht über die (vermutliche) Verbreitung, auf welcher Grundlage lässt sich eine solide Relevanz einer Konzeption wie dem Selbstmanagement-Ansatz begründen? Vordergründig mag dies aus dem Umstand abgeleitet werden, dass bei aktuellen Umfragen zu konkreten Coachinganlässen bei Personalmanagern und Klienten (www.coaching-report.de, 2014) Themen wie die Verbesse-

Dr. K. Kubowitsch (als Gastherausgeber) $(\square)$

Zur Schönen Aussicht 22, 93051 Regensburg, Deutschland

E-Mail: karl.kubowitsch@gap-web.de 
rung sozialer Kompetenz oder eine allgemeine Erweiterung des Verhaltensrepertoires häufig genannt werden. Daneben lässt sich für Coaching im Rahmen von systematischer Personalentwicklung darauf verweisen, dass Ziele und Schwerpunktthemen aus Ergebnissen betrieblicher Instrumente der Personalbeurteilung und Potenzialeinschätzung abgeleitet und schon deshalb primär auf der Ebene konkreter Verhaltensweisen und beobachtbarer Korrelate persönlicher Einstellungen formuliert werden. Ohne anderen Coachingorientierungen die Nähe hierzu absprechen zu wollen, kann für den Selbstmanagement-Ansatz zumindest eine besondere Kompatibilität mit solchen organisationalen Systemen reklamiert werden. In theoretischer Hinsicht schließlich kann man auf die in der therapeutischen wie auch der coachingbezogenen Konzeption postulierte enge Verzahnung mit der gesamten empirischen Psychologie verweisen, um die Relevanz zu untermauern.

Welche Perspektiven mögen sich im Coaching für den Selbstmanagement-Ansatz und seine „Verwandten“ aus dem verhaltensorientierten Spektrum ergeben? Zumindest im angelsächsischen Raum erfahren Coaching-Maßnahmen auf kognitiv-verhaltensorientierter Grundlage eine breite Rezeption; beispielhaft sei hier auf das Handbuch von Palmer und Whybrow (2008) verwiesen. In der Coaching-Praxis dürfte letztlich die Wirksamkeit in Feldern den Ausschlag geben, für die sich dieser Ansatz besonders anbietet: zur nachhaltigen Verbesserung von Leistung und persönlichen Leistungsvoraussetzungen im Management und in High Performance Teams in Organisationen; in typischen Hochrisiko- und Topleistungsbereichen wie der Luftfahrt und bei Einsatzkräften; oder im Spitzensport, für Einzelathleten, Mannschaften und Trainer. Ein Teil dieses Spektrums wird mit den Beiträgen in diesem Heft beleuchtet.

Die Grundlagen des Selbstmanagement-Ansatzes stellt Jörg Pscherer vor. Er skizziert in seinem Beitrag zudem aktuelle Entwicklungen, einschließlich sogenannter neuer „Wellen“ in der Verhaltenstherapie und der Integration von Konzepten der lösungsorientierten Positiven Psychologie. Eine wichtige Zielgruppe für Coaching sind Führungskräfte, zumal bei Maßnahmen im Rahmen betrieblicher Personalentwicklung (www.coaching-report.de, 2014). Als akzeptierter konzeptioneller Rahmen für das Verständnis von Führung kann derzeit der „Full Range of Leadership-Ansatz“ bezeichnet werden. Mahena Stief und Simone Kubowitsch betten selbstmanagementorientiertes Coaching in dieses Modell ein und erläutern praktische Vorgehensweisen anhand von Fallbeispielen. Verhaltenspsychologische Ansätze beziehen traditionell die psychophysiologische Ebene ein. Einen konzeptionellen Rahmen, wie dies für Diagnostik, Intervention und Evaluation im Coaching systematisch zugrunde gelegt werden kann, stellen Simone und Karl Kubowitsch in ihrem Beitrag über Neurocoaching vor. Beispielhaft für das Verständnis von Coaching als Selbstmanagement-Optimierung werden theoretische Aspekte diskutiert und Methoden für die Coaching-Praxis dargelegt.

In den beiden Praxisberichten werden weitere Perspektiven erörtert: Unter dem provokanten Motto „Störfaktor Mensch?!“ diskutiert Ansgar Peiß die Bedeutung von Einstellungen und Verhaltensweisen der an industriellen Großprojekten beteiligten Personen. Für die unterschiedlichen Phasen solcher komplexer Vorhaben werden aus der Sicht eines Auftraggebers aus dem Top-Management die Notwendigkeiten und Voraussetzungen für effektives Coaching erläutert. Die Einbettung von neuartigen Lernumgebungen in Coaching auf konstruktivistischer und instruktionspsychologi- 
scher Grundlage stellen schließlich Thomas Haimerl, Markus Eschbach und Michaela Hanke vor. Ihr Beitrag zeigt, dass in den Selbstmanagement-Ansatz eine breite Palette von Methoden und Erfahrungsräumen integriert werden kann und es sich auch in diesem Sinn um eine offene Konzeption handelt.

\section{Literatur}

Palmer, S., \& Whybrow, A. (2008). Handbook of coaching psychology. Hove: Brunner-Routledge. Schreyögg, A. (2012). Coaching: Eine Einführung für Praxis und Ausbildung (7., überarb. u. erw. Aufl.). Frankfurt a. M.: Campus.

Stahl, G., \& Marlinghaus, M. (2000). Coaching von Führungskräften: Anlässe, Methoden, Erfolg. ZfO, 4, 199-207.

http://www.coaching-index.de/suche. Zugegriffen: 16. Dez. 2014.

http://www.coaching-report.de/definition-coaching/coaching-ablauf/anlaesse-fuer-coaching. Zugegriffen: 16. Dez. 2014.

In der Rubrik „Diskurs“ stehen drei weitere Beiträge zu anderen Themen: Marischa Broermann greift die Debatte um die Professionalisierung in Beratungsberufen auf und erörtert am Beispiel der Erziehungsberatung den Stand der Dinge. Die Etablierung einer Beratungsprofession verlangt - so ihre Schlussfolgerung - die Entwicklung einer akademischen Disziplin Beratungswissenschaft, und zwar nach einem neuen Wissenschaftsverständnis, das dem Selbstverständnis von Beratung in seiner gesamten Heterogenität und den inter- und transdisziplinären Zugängen gerecht wird. Eine Auseinandersetzung mit dem Wissenschaftsverständnis führt auch Thomas Bachmann, und zwar für den Kontext des Coachings. Er polemisiert zum einen gegen die Vorstellung, man könnte Coachinginterventionen mit dem Verabreichen eines Medikaments vergleichen und nach einem solchen Modell empirisch beforschen. Zum anderen plädiert er für eine bessere Zusammenarbeit zwischen Coachingpraktikern und Coachingforschern, damit Forschung die „Anforderungen an Heterogenität, Komplexität und Unschärfe“" aufgreifen kann und auf diese Weise mehr Relevanz für die Coachingpraxis erlangen kann. Auch dies sind maßgebliche Aspekte in der Professionalisierungsdebatte im Coaching. Im dritten Diskursbeitrag von Astrid Schreyögg geht es um die aktuelle Diskussion um die Frauenquote. Anhand einer Studie zur Pfadforschung, in der die Erfahrungen von Managerinnen analysiert wurden, argumentiert die Autorin, dass insbesondere männliche Homogenitätsideologien in Unternehmen einen Erfolg von Frauen im Top-Management erschweren oder verhindern. Wenn die neuen Quotenregelungen gelingen sollen, müssten Firmen ihre Fixierung auf Homogenität einer Revision unterziehen. Auch hier wird wie in den beiden voranstehenden Beiträgen deutlich, dass ein Abschied von traditionsreichen Homogenitätsvorstellungen in Denken und Handeln, in Forschung und Praxis notwendig ist, um der heterogenen Vielfalt in Unternehmen und Organisationen gerecht zu werden und damit dauerhaft zukunftsfähig sein zu können.

Christoph Schmidt-Lellek 